\title{
Künstliche Implantate für die Regeneration peripherer Nerven
}

\author{
Isabell Koxholt und Jörg Mey
}

\begin{abstract}
Zusammenfassung
Da im peripheren Nervensystem axonale Regeneration möglich ist, kann man Verletzungen durch Vernähen durchtrennter Nerven oder durch Nerventransplantationen heilen. Für Transplantationen verwendet man sensorische Nerven, z.B. den $N$. suralis, weshalb an der Entnahmestelle ein sensorischer Funktionsverlust entsteht. Folglich besteht ein medizinischer Bedarf an künstlichen Nervenimplantaten. Diese müssen axonale Regeneration und die Migration von Schwannzellen fördern und dürfen natürlich keine Entzündungsreaktion hervorrufen. Bereits heute werden leere Röhren zur Verbindung von Nervenstümpfen bei Menschen eingesetzt. Allerdings ist es nicht möglich, mit diesen Brücken Distanzen größer als $30 \mathrm{~mm}$ durch Regeneration zu überwinden. Um das zu erreichen, wurden eine Reihe natürlicher und synthetischer Materialien getestet und verschiedene Konstruktionsstrategien erprobt. Man verwendet biokompatible Röhren, die interne Leitstrukturen enthalten. Dafür werden parallele Fasern durch Elektrospinnen hergestellt, oder man produziert longitudinale Kanäle durch Gefriertrocknung von Gelen. Daneben werden Implantatmaterialien mit biochemischen Funktionalitäten versehen. Dies sind vor allem Proteine der extrazellulären Matrix oder kurze synthetische Peptide, die zelluläre Integrine aktivieren. Andere Ansätze verwenden Gradienten neurotropher Faktoren oder inkorporieren regenerationsfördernde Zellen. Fernziel der Forschung ist jedoch die Entwicklung zellfreier künstlicher Nervenbrücken, die genau so gute Regeneration ermöglichen, wie sie in autologen Nerventransplantaten möglich ist.
\end{abstract}

\begin{abstract}
Artificial implants for peripheral nerve regeneration.

Axonal regeneration is possible in the peripheral nervous system. Therefore, nerve lesions can be cured by suturing the dissociated nerve stumps or by grafting an autologous nerve. Since nerve transplantations cause a sensory deficit at the donor site it is desirable to develop artificial implants for nerve regeneration. Artificial implants have to promote and guide axonal growth, the migration of Schwann cells and must not cause inflammation. Hollow tubes as nerve bridges are already used in the clinic. However, with these it is not possible to achieve nerve regeneration over distances much larger than $30 \mathrm{~mm}$. For this purpose, a number of natural and synthetic materials have already been tested. Biocompatible tubes are being developed which contain orientated fibers or gels with longitudinal channels. In addition, artificial guidance materials are endowed with specific biological functions. Most frequently, extracellular matrix proteins or synthetic peptides that activate integrin receptors are coupled to the materials. Other approaches use gradients of neurotrophins or incorporate living cells. In the long run, a major goal of research is to develop cell-free artificial implants which allow a similar degree of regeneration as is possible with autologous nerve transplants.
\end{abstract}

Keywords: peripheral nerve; regeneration; biomaterials; electrospinning; artificial implants

\section{Einleitung}

Periphere Nerven steuern unsere Muskeln und übermitteln Sinneseindrücke an das zentrale Nervensystem. Wenn bei Verletzungen des Gesichts oder der Gliedmaßen periphere Nerven zerstört werden, fallen natürlich auch diese Funktionen aus. Im Unterschied $\mathrm{zu}$ den überwiegend degenerativen Veränderungen nach Rückenmarksverletzungen können Axone im peripheren Nervensystem (PNS) regenerieren. Die Zellkörper dieser Neurone befinden sich nicht in der Peripherie sondern im Vorderhorn des Rückenmarks selbst (Motoneurone), in den Dorsal- wurzelganglien auf beiden Seiten des Rückenmarks (sensorische Neurone), oder in den Grenzstrangganglien, ebenfalls in der Nähe des Rückenmarks (Nervenzellen des vegetativen Nervensystems). Bei der Innervation z.B. des Gesichts durch die Hirnnerven findet man Nervenzellen in Kernen des Hirnstamms. Wenn also Fasern in peripheren Nerven durchtrennt werden, sind die Zellen in der Lage, ein Wachstumsprogramm zu aktivieren, so dass ihre Axone in geeigneter Umgebung regenerieren können. Deshalb kann man nach Nervenverletzungen die beiden Nervenenden wieder miteinander vernähen. Ist der Nerv jedoch nicht glatt durchtrennt worden oder ist die Lücke zwischen den beiden Nervenenden zu groß, setzen Chirurgen Nerven ein, die sie einer anderen Stelle des Patienten entnehmen (autologe Nerventranspantate). Für die Regeneration von Axonen sind verletzte periphere Nerven tatsächlich das beste Material, das man kennt. Weil dadurch jedoch die Funktion des Spendernervs ausfällt, sucht man seit über 25 Jahren nach Alternativen für autologe Transplantate. Einerseits wurden nicht-autologe, azelluläre Transplantate verwendet, andererseits künstliche Implantate aus natürlichen oder synthetisch hergestellten Materialien (Exkurs 1).

\section{Regeneration im peripheren Nervensystem}

Ein Blick auf die Vorgänge während der peripheren Nervenregeneration zeigt, welche Anforderungen künstliche Implantate erfüllen müssen, um autologe Transplantate ersetzen zu können (Abbildung 1). Nach Verletzung eines peripheren Nervs kommt es zunächst zu der so genannten Wallerschen Degeneration im distalen Teil des Nervenstumpfes. Da die Axone vom Zellkörper getrennt wurden, sind sie auf Dauer nicht lebensfähig. Das Zytoskelett der Neuriten wird abgebaut, die Zellmembranen lösen sich auf, und die Schwannzellen verlieren ihre Myelinschicht. Makrophagen entfernen Bruchstücke des Myelins und der degenerierenden Fasern. Gleichzeitig werden von den Axonen im proximalen Nervenstumpf Wachstumskegel gebildet. In einer wachstumspermissiven Umgebung, das heißt, wenn eine Verbindung zum degenerierenden distalen Nerv besteht, wachsen die Axone entlang longitudinaler Strukturen aus proliferierenden Schwannzellen und Perineurium, den sogenannten Büngerschen Bändern (Sulaiman et al. 2005). Die Regeneration erreicht Geschwindigkeiten von einigen 
Millimetern pro Tag. Verletzte periphere Nerven, vor allem die von den Schwannzellen gebildete Basallamina, stellen das beste bekannte Wachstumssubstrat für die axonale Regeneration dar. Anschließend myelinisieren Schwannzellen erneut die Axone. Das axonale Wachstum wird von zahlreichen parakrin sezernierten Faktoren stimuliert, die vor allem von den Schwannzellen abgegeben werden (Boyd und Gordon 2003). Wenn Axone am Zielorgan ankommen, können sie neue Synapsen und Endorgane ausbilden, so dass der Nerv wieder voll funktionsfähig sein kann.

\section{Anforderungen an künstliche Nervenimplantate}

Die Beschreibung macht deutlich, welche Funktionen künstliche Implantate $\mathrm{zu}$ erfüllen haben, wenn sie funktionelle Nervenregeneration in vivo erlauben sollen (Dalton und Mey 2009). Zunächst muss das implantierte Konstrukt grundsätzlich biokompatibel sein. Das bedeutet, dass keine Entzündungsreaktionen ausgelöst werden, dass das Implantat nicht in einer Narbe abgekapselt und nicht abgestoßen wird. Neben biochemischen spielen hier mechanische Eigenschaften eine Rolle, weil z.B. bei zu geringer Elastizität die Reizung des Gewebes Entzündung hervorrufen kann. Quellung des Implantats würde das Lumen verringern und regenerierende Fasern quetschen (de Ruiter et al. 2009). Es ist außerdem erwünscht, dass die Brücke zwischen zwei Nervenstümpfen solange im Körper verbleibt, wie es für die axonale Regeneration nötig ist. Das Material soll dann aber allmählich abgebaut werden, wobei keine toxischen Abbauprodukte entstehen dürfen. Die implantierte Struktur muss permeabel sein, um die Diffusion von Atemgasen, Nährstoffen und biologischen Signalmolekülen wie Hormonen und Neurotrophinen zuzulassen. Dazu sollten die Blutgefäße einwachsen, die auch einen normalen Nerv versorgen. Die zentrale Funktion besteht natürlich darin, regenerierende Axone vom proximalen zum distalen, peripheren Nervenstumpf zu leiten. Dazu müssen die axonalen Wachstumskegel physische Leitstrukturen erkennen, die ihrerseits durch Aktivierung bestimmter Rezeptoren der Zellen deren gerichtetes Wachstum fördern. Ähnliche molekulare Interaktionen sollten auch die Migration von Schwannzellen ermöglichen. Schwannzellen sind unbedingt notwendig, nicht nur für die Regeneration der verletzten Axone, sondern wegen der Myelinisierung auch für ihre spätere Funktion.

\section{Konstruktionsstategien für Nervenbrücken}

Leere Röhren aus verschiedenen Materialien.

Implantate, die aus leeren Röhren bestehen, wurden bisher im Tierversuch am häufigsten verwendet. Die Röhren können aus natürlichen oder aus synthetischen Materialien bestehen (Exkurs 1). Im einfachsten Fall handelt es sich um eine leere Röhre, die nur aus einem Material angefertigt ist, z.B. Chitosan, Collagen (natürliche Materialien) oder Silikon, PLA, PGLA und PCL (synthetische Materialien, Exkurs 2). Leere Röhren wurden bereits in klinischen Studien getestet (Meek und Coert 2008). In Tierversuchen wurden Implantate hauptsächlich in Ischiasnerven von Ratten eingesetzt. Bei diesen Experimenten betragen die Nervenläsionen selten mehr als $20 \mathrm{~mm}$, wobei die Versuche mit leeren Röhren meist nur die Grundlage für weitere Experimente sind. So werden die Biokompatibilität und das Potenzial des ausgewählten Materials untersucht, als Brücken bei peripheren Nervenverletzungen zu dienen. Die
Lumen dieser Röhren können dann mit Gelen, Fasern oder Gerüsten strukturiert oder das Material mit biochemisch aktiven Faktoren modifiziert werden.

\section{Strukturierte Implantate: Gele, Fasern und Gerüste.}

Mechanische Leitstrukturen im Innern künstlicher Röhren sollten die periphere Nervenregeneration verbessern, indem sie den Nervenfasern einen Halt geben und sie in eine bestimmte Richtung lenken. Die Konstruktion von Leitstrukturen im Implantat stellt allerdings nach wie vor eine technische Herausforderung dar (Abbildung 2). Unterschiedlichen Strategien werden am Beispiel künstlicher Nano- und Mikrofasern ersichtlich. Mit der Methode des Elektrospinnens können synthetische Polymere zu parallelen Fasern verarbeitet werden, die mit $100 \mathrm{~nm}$ bis $5 \mu \mathrm{m}$ Durchmesser sehr dünn sind und deshalb eine verhältnismäßig große Oberfläche besitzen. Diese dienen migrierenden Schwannzellen und regenerierenden Axonen als Wachstumssubstrat und bieten sich zur Strukturierung künstlicher Implantate an (Schnell et al. 2007). In einem Ansatz wurden Mikrofasern aus Polyglycolsäure in eine Chitosanröhre eingelagert. Das Implantat wurde in $30 \mathrm{~mm}$ lange Lücken in den Ischiasnerv von Beagles eingesetzt und nach sechs Monaten untersucht. Die Hunde, die das Implantat erhalten hatten, erlangten die Funktion des verletzten Nervs wieder, und der Skelettmuskel konnte reinnerviert werden. Das Implantat war innerhalb dieses halben Jahres vollständig abgebaut (Wang et al. 2005). In einem anderen Fall wurden aus elektrogesponnenen Poly(acrylnitril-co-methylacrylat) Faserfilme

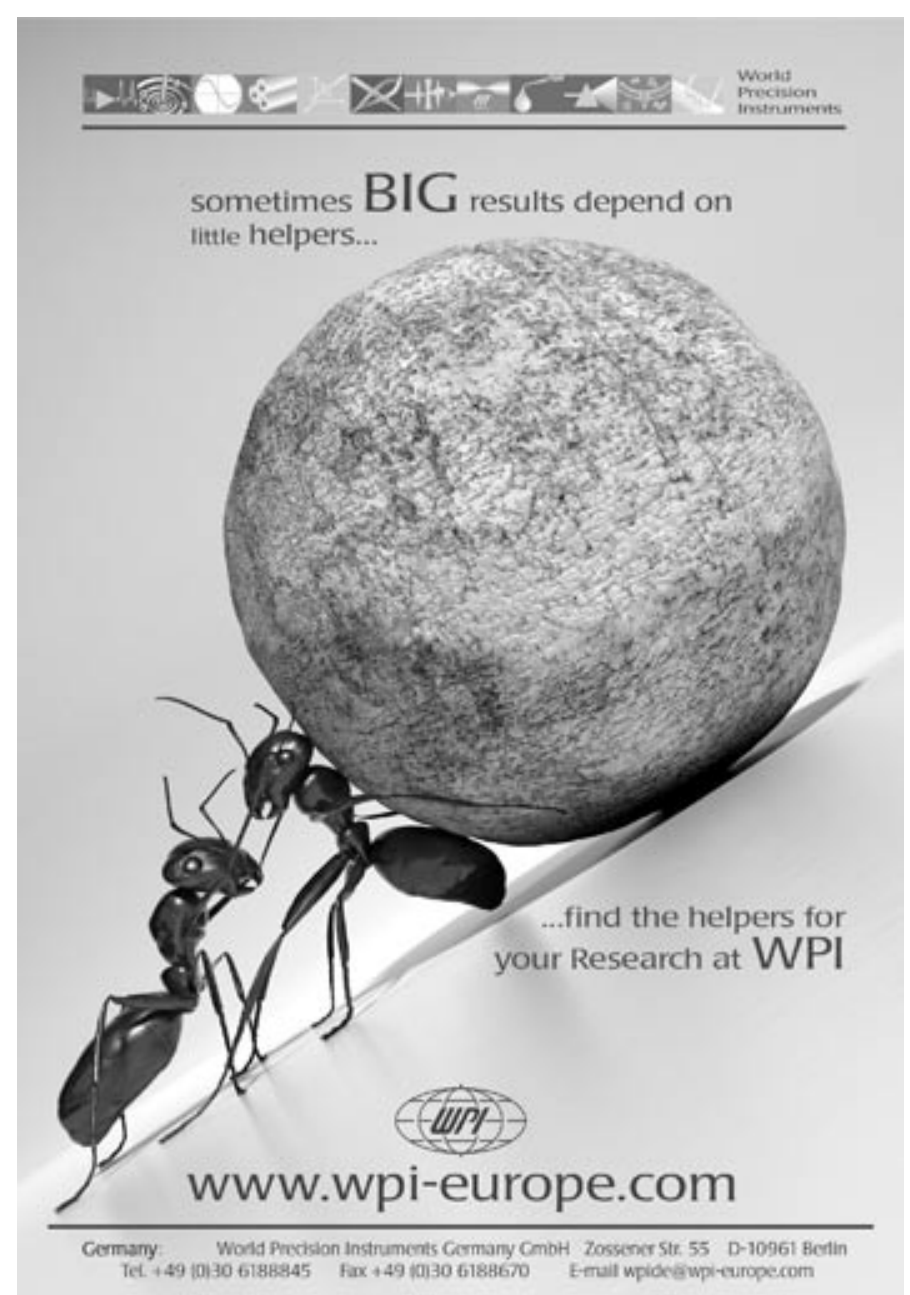




\section{Exkurs 1}

\section{Nervenimplantate}

Autologe Nerventransplantate bilden den Goldstandard der Therapie von Nervenverletzungen beim Menschen. Nachteile sind Notwendigkeit einer zweiten Operation und Funktionsverlust an der Entnahmestelle.

Allogene Nerventransplantate stammen aus einem anderen Individuum der gleichen Art, Xenotransplantate aus artfremdem Gewebe. Immunsuppression ist notwendig. Durch thermische, chemische Behandlung oder radioaktive Bestrahlung können Zellen abgetötet werden, wobei die extrazellulären Matrix (EZM) erhalten bleibt.

Transplantate anderer Gewebetypen wurden als Alternativen erprobt, z.B. Muskelfasern, Sehnen, Blutgefäße, Submukosa des Dünndarms.
Künstliche Implantate können aus natürlichen Materialien bestehen. Am häufigsten kommen Proteine der EZM zum Einsatz, insbesondere Laminin, Collagen und Fibronectin. Andere natürliche Moleküle für die Konstruktion von Nervenbrücken sind Hyaluronsäure, Fibrin, sowie Polysaccharide Agarose, Alginat und Chitosan (deazetyliertes Chitin). Durch Quervernetzungstechniken können Strukturen länger haltbar gemacht werden. Meist werden verschiedene Substanzen kombiniert und zu longitudinal ausgerichteten Fibrillen verarbeitet.

Künstliche Implantate, die aus synthetischen Materialien bestehen, sind für die Reparatur peripherer Nervenverletzungen interessant geworden, weil man ihre chemischen und physikalischen Eigenschaften gut kontrollieren kann. Dabei stellen Biokompabilität und biologische Abbaubarkeit Herausforderungen dar. Die wichtigsten Materialien sind in Exkurs 2 genannt, Strukturformeln in Abbildung 3A.

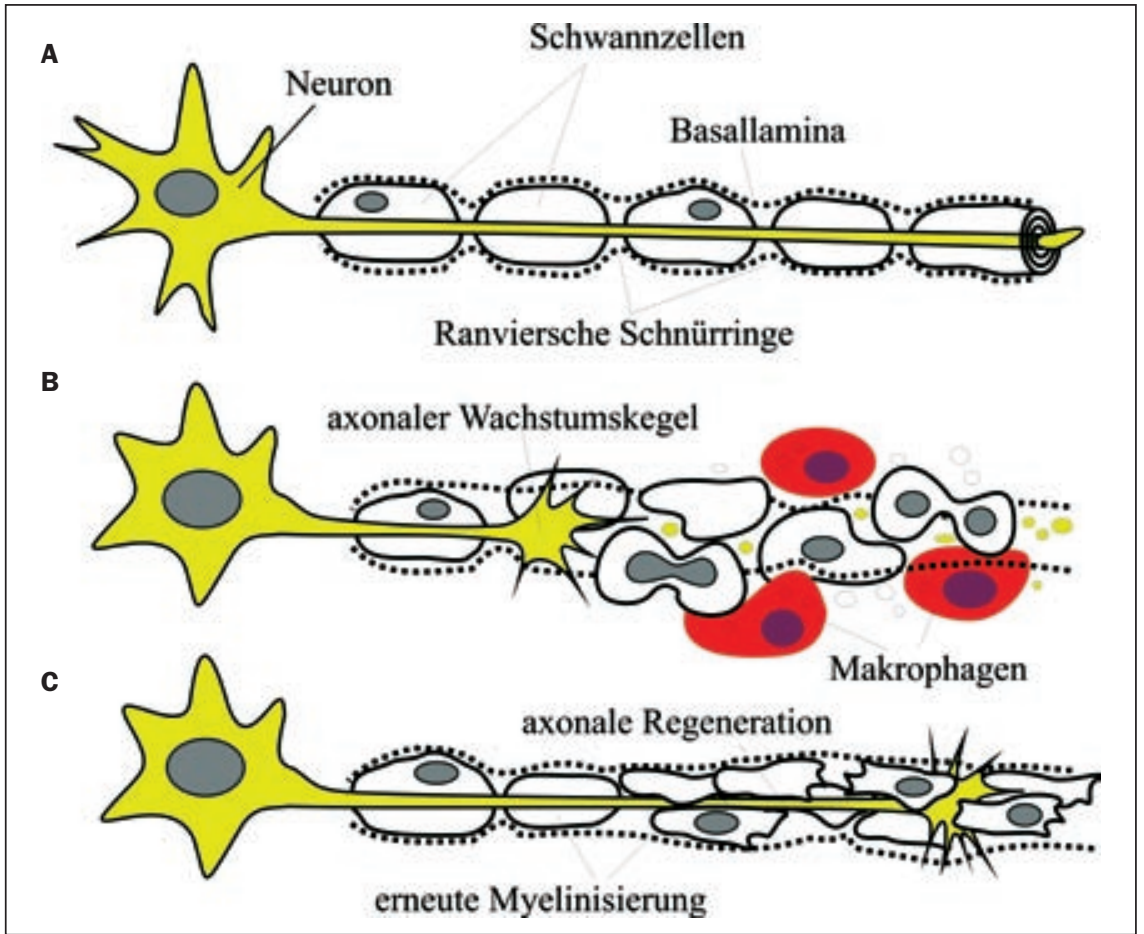

Abb. 1: Regeneration im peripheren Nerven. A: Die meisten Neurone, deren Axone in peripheren Nerven verlaufen, befinden sich im ventralen Rückenmark (Motoneurone), den Dorsalwurzelganglien (sensorische Nervenzellen) oder den Grenzstrangganglien (Neurone des vegetativen Nervensystems). Axone sind vom Myelin der Schwannzellen umhüllt. B: Nach einer Läsion degenerieren Myelinhüllen und Axone distal der Verletzungsstelle. Schwannzellen proliferieren, Makrophagen entfernen degenerierende Fasern. C: An der Verletzungsstelle bilden Neurone neue axonale Wachstumskegel aus, und Axone können entlang der longitudinal angeordneten Schwannzellen und EZM regenerieren. Schwannzellen myelinisieren die neuen Axone.

gestapelt und in eine Polysulfanröhre eingefügt. Diese Implantate wurden in verletzte Ischiasnerven von Ratten eingesetzt, um einen Defekt von $17 \mathrm{~mm}$ zu überbrücken. Sechzehn Wochen nach der Operation war der Zielmuskel erneut innerviert, und die Untersuchungen zeigten eine Regeneration der sensorischen und motorischen Nervenfasern (Kim et al. 2008). In einer anderen Studie wurden Faserfilme aus Poly(acrylnitril-co-methylacrylat) so in eine Röhre eingefügt, dass man eine Zförmige Unterteilung des Röhrenlumens erhielt (Abbildung 2D). Im Ischiasnerv von Ratten konnten nach drei Monaten Nervendefekte von $14 \mathrm{~mm}$ überbrückt werden (Clements et al. 2009).

Eine Alternative zu elektrogesponnenen Fasern besteht darin, Gerüste mit kanalähnlichen Leitstrukturen herzustellen (Abbildung 2C). Ein erfolgversprechendes Verfahren hierfür ist die gerichtete Gefriertrocknung, bei der fingerförmige Eiskristalle in einer Collagendispersion oder Lösung wachsen. Dabei wird das Collagen konzentriert und erzeugt so die Wände orientierter Hohlräume. Mit dieser Methode ist es gelungen, dreidimensionale Konstrukte mit Schwannzellen zu besiedeln (Bozkurt et al. 2009), die nun in Tierversuchen getestet werden.

Die Entwicklung geeigneter physikalischer Strukturen ist wahrscheinlich nicht ausreichend, um künstliche Implantate $\mathrm{zu}$ erhalten, die ähnlich geeignet sind wie autologe Nerventransplantate. Dafür ist die chemische Funktionalisierung des Materials mit biologischen Signalmolekülen wahrscheinlich eine weitere Voraussetzung.

\section{Funktionalisierung mit Molekülen der extrazellulären Matrix}

Bei der Funktionalisierung geht es darum, durch das implantierte Material körpereigene Zellen (Neurone, Schwannzellen, Endothelzellen) so zu aktivieren, dass die Nervenregeneration gefördert wird (Abbildung 3B-D). Man kann grob zwei Strategien unterscheiden, nämlich die Anlagerung von Molekülen der extrazellulären Matrix (EZM) und die kontrollierte Freisetzung von Wachstumsfaktoren.

Ein nahe liegender Ansatz besteht in der Verwendung von EZM-Molekülen, weil Axone natürlicherweise entlang der von Schwannzellen gebildeten Basallamina wachsen. Proteine oder Peptide werden mit synthetischen Polymeren verbunden. Dies geschieht entweder durch Mischung, Adsorption oder kovalente Bindung (Koh 
et al. 2008). Durch diese Funktionalisierung sollen körpereigene Zellen in das Implantat einwandern und so größere Nervenläsionen schließen. Laminine, die Hauptbestandteile der Basallamina, werden dabei am häufigsten verwendet, zumal Laminin-Beschichtungen schon lange zur Modifizierung von Zellkulturschalen verwendet werden. Auch Collagen in Mischungen mit synthetischen Polymeren hat sich als geeignet erwiesen (Schnell et al. 2007). Neben der Verwendung ganzer EZM-Proteine, die vorher extrahiert wurden (Matsumoto et al. 2000), gibt es auch die Strategie, kurze Peptide aus Laminin, Fibronectin oder Collagen zu benutzen (Dalton und Mey 2009; Wang et al. 2008). Hierbei nutzt man die Kenntnis spezifischer Aminosäurensequenzen in der EZM, die Integrin-Rezeptoren auf Schwann- und Nervenzellen aktivieren.

Diese Sequenzen sind nicht nur wegen der intrazellulären Ankopplung von Integrinen an das Zytoskelett adhäsiv, sie aktivieren auch vielfältige physiologische Signalkaskaden im Innern der Zellen (Geiger et al. 2001). Erfolgreich eingesetzt wurden bisher Peptide mit den Aminosäurensequenzen RGD, YIGSR, IKVAV und verschiedene verlängerte Versionen davon. Im Labor der Autoren wurde zum Beispiel gezeigt, dass die kovalente Bindung von GRGDS an elektrogesponnene PCL-Fasern deren Eignung als Leitstrukturen für axonales Wachstum verbessert (Klinkhammer et al. 2010). Verschiedene Möglichkeiten der Funktionalisierung durch EZM-Moleküle werden derzeit auch in vivo getestet. So wurde eine Chitosanröhre innen mit verschiedenen Peptiden aus Laminin modifiziert. Als Implantat im Ischiasnerv von Ratten förderte eine der gewählten Sequenzen die Nervenregeneration genauso gut wie Allotransplantate, war jedoch körpereigenen Transplantaten noch unterlegen (Wang et al. 2008).

Neben EZM-Proteinen stellen Zelladhäsionsmoleküle (wichtiges Beispiel: L1) eine andere Gruppe oberflächengebundener Signale dar, die das Neuritenwachstum induzieren können. Auch diese Transmembranproteine, die sich also nicht in der EZM sondern auf Zellen befinden, werden zur Funktionalisierung synthetischer Materialien in Betracht gezogen (Webb et al. 2001). Die chemische Funktionalisierung synthetischer Materialien mit Molekülen der EZM war einer der wichtigsten Entwicklungsschritte zu Verbesserung künstlicher Implantate (Ergebnisse: Abbildung 4). Hierbei handelt es sich immer um Signale, die fest

\section{Exkurs 2}

Synthetische Materialien für künstliche Nervenbrücken

Polyhydroxysäuren (Abb. 3A). Für die Nervenregeneration werden Polyhydroxysäuren zu Fasern gesponnen, die auch mit natürlichen Proteinen funktionalisierbar sind. Die wichtigsten sind Polylactid (PLA, Stereoisomere: PLLA, PDLA), Polylactidco-Glycolid (PLGA), Poly( $\varepsilon$-Caprolacton) (PCL) und Polyhydroxybuttersäure (PHB). Fasern und Gewebe finden vielfältige Anwendungen im tissue engineering, z.B. als Gerüst- und Nahtmaterial.

Polyethylenglycol (PEG; Abb. 3A). Aus PEG synthetisiert man das hydrophobe Star-NCO-Polyethylenglykol-stat-Polypropylenglykol (sPEG), einen sternför- migen Polyether mit einem Rückgrat aus $80 \%$ Ethylenoxid und 20\% Propylenoxid, dessen Isozyanat-Endgrupppen zur kovalenten Modifikation anderer Polymere dienen (Abb. 3D).

Hydrogele, z.B. Poly(2-hydroxyethylmethacrylat) (pHEMA) oder Agarose, mit einem hohen Wasseranteil (>90\%) können als Gerüste mit strukturierten Kanälen, zur Aufnahme biologisch aktiver Wachstumsfaktoren oder für die Aufnahme elektrogesponnener Fasern verwendet werden.

Nicht biologisch abbaubare Polymere für Nervenimplantate sind Silikone, Polyurethan, Polytetrafluorethylen (PTFE), Poly(organo)phosphazan. Polypyrrol ist ein leitfähiges Polymer, das zusätzlich elektrische Stimulation regenerierender Nervenfasern erlaubt.

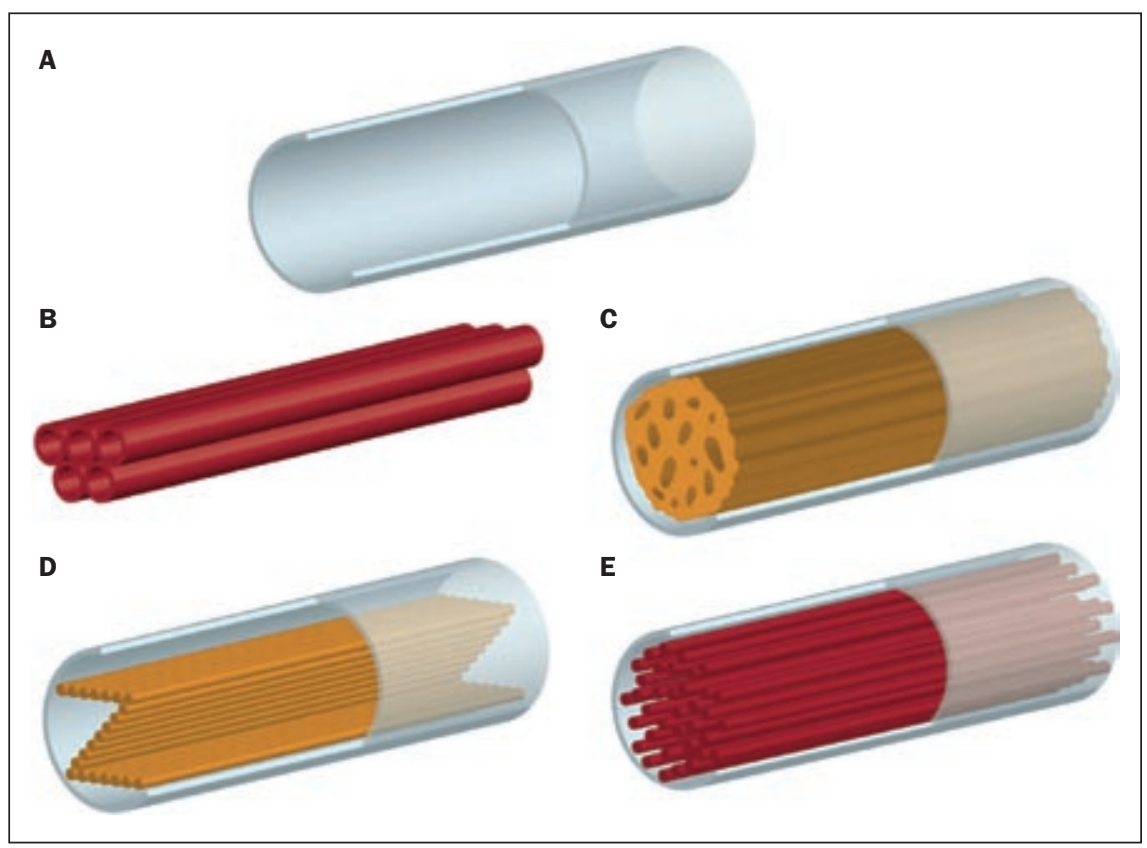

Abb. 2: Konstruktionsstrategien für künstliche Nerventransplantate. A: Leere Röhren zur Verbindung peripherer Nerven bilden die Basis der meisten Implantate. B: Künstliche Nervenbrücke, die aus mehreren zusammen gelagerten hohlen Röhren besteht (Hadlock et al. 1998). C: Durch Gefriertrocknung können Gele mit gerichteten Kanälen hergestellt werden (Bozkurt et al. 2009). D: Elektrogesponnene Mikrofasern auf Filmen sind im Lumen einer Röhre angeordnet (Clements et al. 2009). E: Elektrogesponnene Fasern können auch dreidimensional gesammelt und in Röhren aufgenommen werden (Rumman und Mey, unveröffentlich).

an Oberflächen gebunden sind. Daneben gibt es eine Anzahl kleinerer, in der Regel sezernierter, Faktoren die für das axonale Wachstum bei der Regeneration in einem gequetschten oder transplantierten Nerv von Bedeutung sind.

\section{Kontrollierte Freisetzung von Wachstumsfaktoren}

Einige dieser Faktoren wurden in verschiedenen Studien in vitro und in vivo getestet, teils mit kontrollierter Ausschüttung, teils 


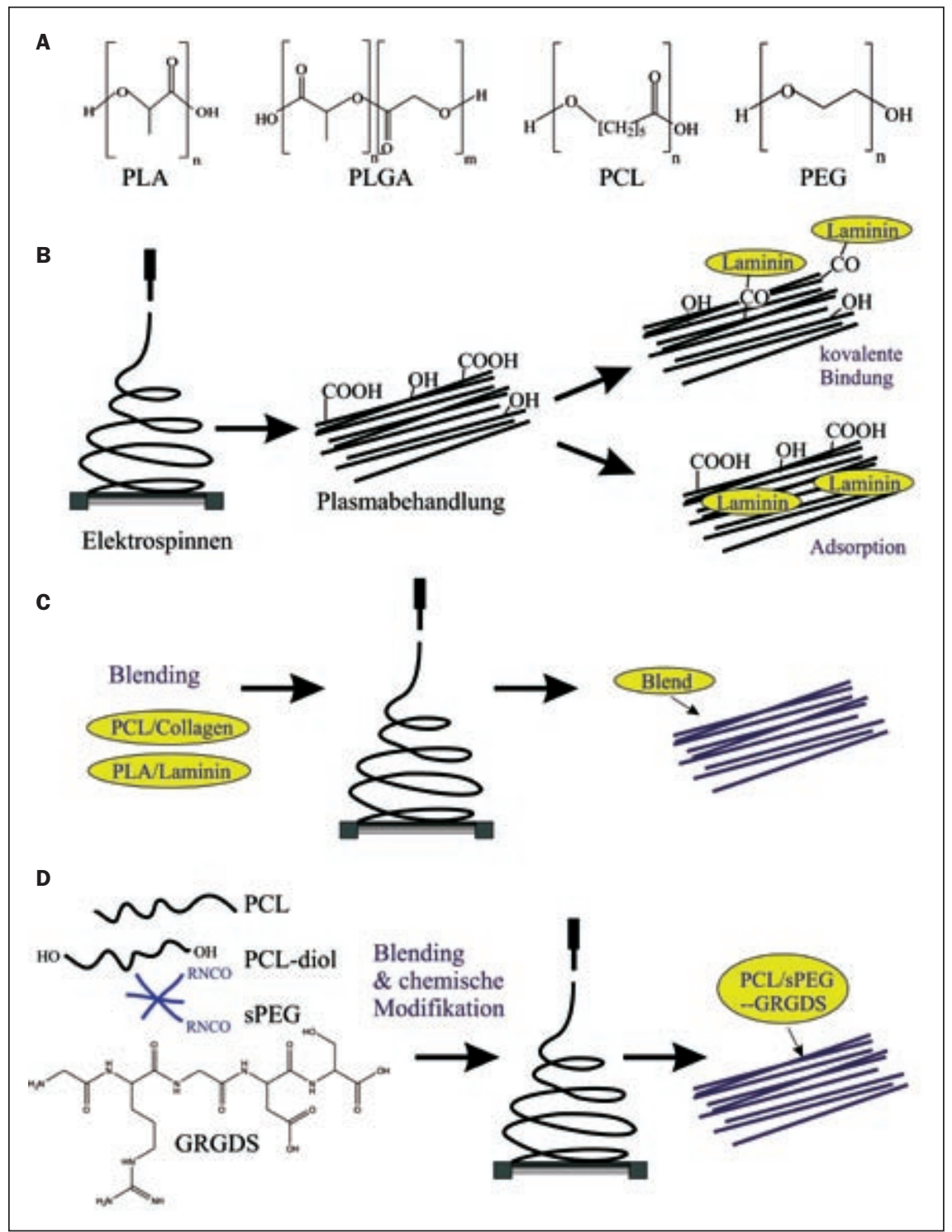

Abb. 3: Funktionalisierung von Leitstrukturen für die Nervenregeneration. A: Strukturformeln synthetischer Polymere, die zur Konstruktion von künstlichen Implantaten verwendet werden (vgl. Exkurs 2); B: Funktionalisierung durch Adsorption oder kovalente Bindung von EZM-Molekülen an elektrogesponnene Polymerfasern (Koh et al. 2008); C: Funktionalisierung durch Mischen (blending) von EZM-Molekülen und Polymerfasern (Schnell et al. 2007); D: Funktionalisierung durch chemische Modifizierung vor dem Elektrospinnen (Klinkhammer et al. 2010).

mit künstlich hergestellten Gradienten. Dafür wurden vor allem zwei Methoden erprobt. Zum einen kann das Implantat mit einer Lösung gefüllt werden, die Wachstumsfaktoren enthält, zum anderen können Carrier-Systeme mit den Wachstumsfaktoren eingeführt werden, die sie dann nach und nach freisetzen. Die letztere Lösung wird bevorzugt, da hier die Freisetzung der Wachstumsfaktoren reguliert und außerdem ein Gradient produziert werden kann, der vermutlich auch im natürlichen Gewebe vorliegt. Verschiedene Probleme müssen gelöst al. 2003). Dies war bislang eine der größten Distanzen, die durch Implantate geschlossen werden konnten. In einer anderen Studie wurde der Wachstumsfaktor glial derived neurotrophic factor (GDNF) von elektrogesponnenen Fasern freigesetzt. Mit diesen Fasern in der Innenwand einer Röhre gelang die funktionelle Regeneration über $16 \mathrm{~mm}$ im Ischiasnerv von Ratten (Chew et al. 2007). In anderen Studien wurden die Funktionalisierung mit Wachstumsfaktoren und EZM-Proteinen kombiniert. So diente beispielsweise ein Agarosegel zur Präsentation von Gradienten an nerve growth factor (NGF) und Laminin. Dabei hatte neben dem NGF auch der LamininGradient einen positiven Effekt auf die Nervenregeneration (Dodla und Bellamkonda 2008). Da es kaum vergleichende Studien der verschiedenen Strategien zur Funktionalisierung gibt, kann zurzeit nicht gesagt werden, welche der Moleküle am besten für periphere Nervenregeneration geeignet ist.

\section{Einschluss von Zellen ins künstliche Implantat}

Den autologen Nerventransplantaten kommen Konstruktionen am nächsten, bei denen regenerationsfördernde Zellen im künstlichen Implantat mit eingebracht werden. Diese Zellen, die körpereigen oder körperfremd sein können, sollen biologische Signale abgeben, die teilweise noch nicht bekannt sind und deren Aktivität durch künstliche Modifizierung kaum nachgeahmt werden kann.

Am besten geeignet hierfür sind Schwannzellen, die natürlicherweise das PNS bevölkern. Neben Schwannzellen kommen vor allem olfaktorische Gliazellen, mesenchymale Stammzellen oder genetisch modifizierte Zellen in Frage (Schmidt und Baier Leach 2003). In der Mehrzahl der Versuche zur peripheren Nervenregeneration wurden Schwannzellen verwendet. Sie unterstützen die Nervenregeneration auf vielfältige Weise, unter anderem weil sie die Büngerschen Bänder bilden, an denen entlang die Axone regenerieren. Zudem synthetisieren Schwannzellen EZM-Moleküle, geben verschiedene Wachstumsfaktoren ab, unter anderem Neurotrophine, CNTF, GDNF und TGF $\beta$ (Boyd und Gordon 2003). Natürlich bringt die Implantation fremder Zellen eine Reihe zusätzlicher Komplikationen mit sich. Zunächst ist die Reinheit der Schwannzellen-Kultur wichtig, da diese nicht mit Fibroblasten kontaminiert sein darf. Dann stellt sich die Frage, aus welcher Quelle die 
Schwannzellen stammen sollen. Während bei Inzuchtstämmen von Ratten und Mäusen die Entnahme aus neugeborenen oder erwachsenen Tieren gut möglich ist, wird das beim Menschen zum Problem. Für die mögliche Therapie von ZNS-Verletzungen gewinnt man bereits jetzt olfaktorische Gliazellen aus der Riechschleimhaut des Menschen (Kawaja et al. 2009). Vielleicht bietet dies eine Möglichkeit auch für die Herstellung von Implantaten für periphere Nerven. Wegen der grundsätzlichen Probleme der Immunabstoßung bei Implantation von fremdem Gewebe scheint uns die Weiterentwicklung zellfreier künstlicher Nervenbrücken wichtig zu sein.

\section{Klinische Studien mit künstlichen Nervenimplantaten}

Es sind bereits mehrere Typen von Implantaten für periphere Nervenregeneration im Menschen zugelassen (Meek und Coert 2008). Diese wurden in mehreren hundert Patienten getestet. NeuroGen besteht aus Typ 1-Collagen und wird von der Firma Integra Neuroscience vertrieben (www. integra.1s.com). In der bislang größten Studie mit insgesamt 96 Patienten wurden 126 Nervenverletzungen vor allem in sensorischen Nerven der Arme überbrückt. Sechsundzwanzig Patienten wurden quantitativ und sechs Patienten qualitativ untersucht, um die funktionelle Wiederherstellung der Nerven zu ermitteln. Bei $45 \%$ aller Patienten wurde eine Verbesserung der sensorischen Funktion festgestellt (Wangensteen und Kalliainen 2009). Neurotube ist ein aus PGA bestehendes Implantat, das von Synovis Life Technologies Inc. produziert wird (www.synovismicro.com). Es wurde in verschiedenen klinischen Studien eingesetzt. Laut einer im Jahr 2000 veröffentlichten Arbeit über PGA-Implantate erhielten 24 Patienten mit Läsionen der Handnerven ein NeurotubeImplantat, einer anderen Gruppe von 74 Patienten wurden körpereigene Nerven transplantiert. Die Nervendefekte betrugen zwischen $2 \mathrm{~mm}$ und $12 \mathrm{~mm}$. Während der Behandlungszeit wurden mit allen Patienten Rehabilitationsmaßnahmen durchgeführt. Bei insgesamt 18 Patienten kam es auf Grund des Implantats zu Komplikationen, jedoch wurden $91 \%$ der Patienten mit PGA-Implantat geheilt, hingegen in der Kontrollgruppe nur 49\% der Patienten (Weber et al. 2000). Das dritte zugelassene Implantat heißt Neurolac und wird von der Firma Polyganics Inc. vertrieben (www. polyganics.com). Es besteht aus einer Mischung von PLA und PCL. Bisher wurden
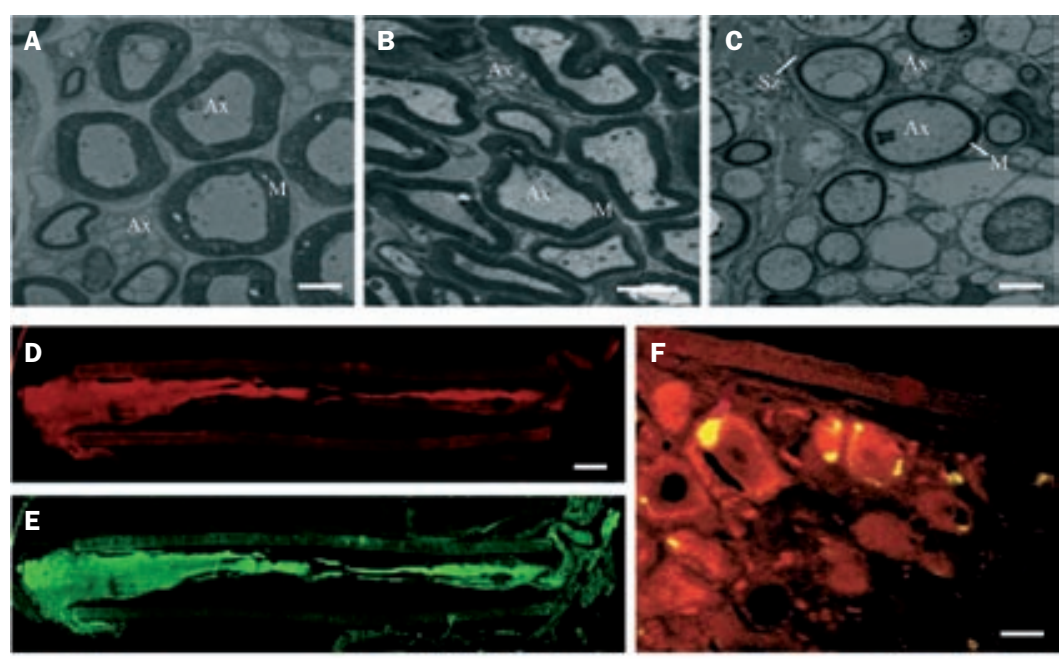

G
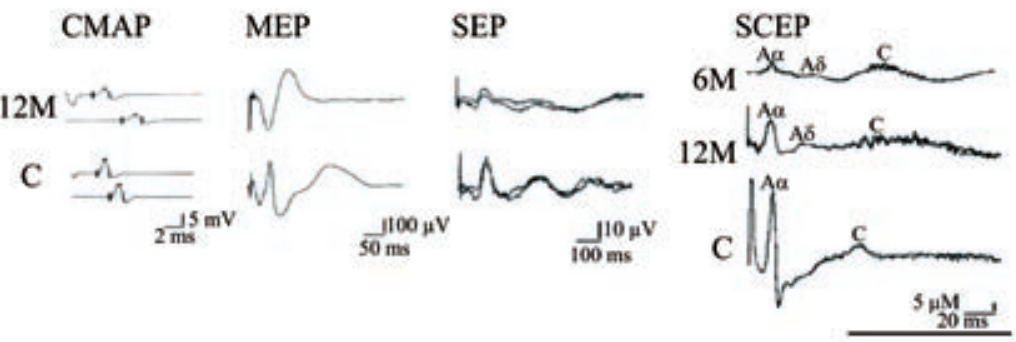

H

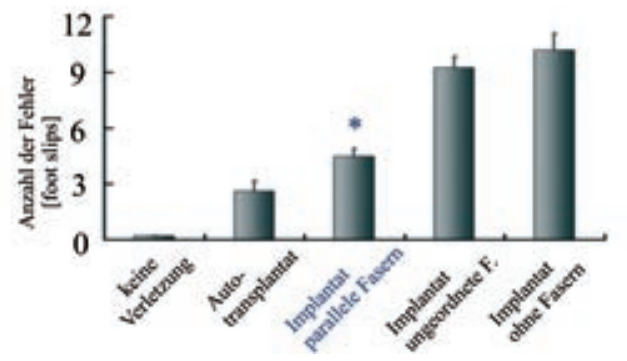

Abb. 4: Experimentelle Ergebnisse mit künstlichen Implantaten. A-C: Elektronenmikroskopische Aufnahmen von Nervenquerschnitten regenerierter Nerven, Maßstab 2 um: A unverletzter Ischiasnerv der Ratte; B: Regeneration durch ein Autotransplantat; c durch ein Implantat mit NGF- und Laminingradienten, Distanz 10 mm, nach vier Monaten; Ax Axone, M Myelinhüllen, Sz Schwannzelle (Dodla und Bellamkonda 2008); D, E: regenerierte Nervenfasern in einem Implantat mit elektrogesponnenen Mikrofasern; Ischiasnerv der Ratte nach vier Monaten, immunhistochemische Färbung von Längsschnitten, oben (rot): Neurofilament, Axone; unten (grün): S100, Schwannzellen (Kim et al. 2008); F: retrogrades FluoroGold-Tracing vom distalen Ende eines Chitosan/PGA-Implantats zeigt axonale Regeneration von Neuronen im Dorsalwurzelganglion, Hunde, sechs Monate nach Implantation (Wang et al. 2005); G: elektrophysiologischer Nachweis der Funktion regenerierter Neurone über eine Lücke von 80 mm, Hunde, nach zwölf Monaten; Collagenimplantate mit Lamininbeschichteten Fasern wurden in den $\mathbf{N}$. peronaeus eingesetzt: Muskelpotenziale (CMAP) des anterioren Tibialismuskels nach Reizung des Ischiasnervs; motorisch evozierte Potenziale (MEP) im M. tibialis nach Stimulation des Motorkortex; und somatosensorisch evozierte Potenziale (SEP) abgeleitet über dem somatosensorischen Kortex nach Muskelstimulation und Ableitung des Rückenmarks (SCEP) nach Stimulation des Nervs distal des Implantats (Matsumoto et al. 2000). H: Funktioneller Nachweis der Regeneration durch die in D,E abgebildeten Implantate bei der Ratte. Im grid walking test wurde die Anzahl der Fehler beim Laufen über ein Gitter gezählt. Implantate mit parallel ausgerichteten Fasern ( *) zeigten bessere Erfolge als Implantate ohne oder mit ungerichteten Fasern, waren aber schlechter als autologe Nerventransplantate (Kim et al. 2008). 
zwei klinische Studien veröffentlicht, in denen insgesamt 36 Patienten mit Neurolac behandelt wurden. In einer ersten Studie aus dem Jahr 2003 konnte keine Nervenregeneration festgestellt werden, in einer zweiten war die funktionelle Wiederherstellung der verletzten Nerven genauso gut wie in der Kontrollgruppe (Bertleff et al. 2005).

In Japan werden ebenfalls PGA-Implantate verwendet, die außerdem mit Collagen gefüllt sind, allerdings bei wenigen Patienten. In der neuesten Fallstudie aus dem Jahr 2007 wurden zwei Patienten vorgestellt, die nach Verletzungen von Gesichtsnerven exzellente Heilungsfortschritte erfuhren (Inada et al. 2007). Dieses Implantat wird jedoch nur in Japan verwendet, da es weder in den USA noch in Europa zugelassen ist.

\section{Probleme und Perspektiven}

Im Gegensatz zu Verbindungen im ZNS, wie Rückenmark oder Sehnerv, bieten periphere Nerven bereits heute ein Erfolg versprechendes Arbeitsfeld für neuronales tissue engineering. Zahlreiche biokompatible Materialien sind entwickelt worden, die großes Potenzial für Therapien im Nervensystem haben. Gleichwohl lassen sich verschiedene Problembereiche identifizieren:

Standardisierung der Erfolgskontrolle. Zahlreiche Experimente verschiedener Biomaterialien sind nicht kompatibel, weil ganz unterschiedliche Zellkulturen, Tierversuche und Kriterien des Regenerationserfolgs verwendet wurden. Überhaupt ist ein großer Anteil der Arbeiten, die innovative Biomaterialien beschreiben, hinsichtlich der biologischen Untersuchungsmethoden recht dürftig. Entzündungsreaktionen, Effekte auf Genexpression und Langzeitwirkungen in vivo wurden kaum untersucht. Systematische Vergleiche verschiedener Materialien für künstliche Nervenimplantate sind nötig.

Strukturierte dreidimensionale Implantate. Bisher verwendeten die meisten Studien, bei denen tatsächlich Regeneration in vivo untersucht wurde, nur leere Röhren, in denen Axone zunächst auf der inneren Oberfläche des Implantats wuchsen. Eine technische Herausforderung ist daher die Konstruktion von dreidimensionalen Nervenbrücken mit gerichteten internen Strukturen zur Leitung der axonalen Regeneration und der Migration der körpereigenen Schwannzellen. Interessante Ansätze dazu sind die Einbettung parallel ausgerichteter Mikrofasern (Kim et al. 2008; Lietz et al. 2006) und die Herstellung von Kanälen in Collagenschwämmchen (Bozkurt et al. 2009).

Biomimetische Funktionalisierung von Implantaten. Mit der Verwendung von Proteinen und Peptiden der EZM ahmen künstliche Nervenimplantate schon jetzt Signale natürlicher Nerven nach. Die Anwendung spezifischer Faktoren, die Glia- und Nervenzellen aktivieren, steckt dagegen noch in der Anfangsphase. Neben der Auswahl der molekularen Signale stellen die erforderlichen zeitlichen und räumlichen Aktivitätsmuster eine Herausforderung dar. Neurotrophe Signale könnten z.B. noch nach Monaten und in ansteigenden Gradienten zum distalen Ende eines Implantats nötig sein.

Dreißig-Millimeter-Marke: Die meisten Tierversuche zeigen, dass die Nervenregeneration in den vorhandenen künstlichen Implantaten nur Distanzen von bis zu 30 mm überbrückt. Da für Anwendungen am Menschen auch längere Lücken zu ersetzen sind, wird der Nutzen künstlicher Implantate daran zu messen sein, ob dies erreicht wird. Experimente mit dem Ischiasnerv der Ratte stoßen hier an ihre Grenze. Wir sind überzeugt, dass Erkenntnisse der neurobiologischen Grundlagenforschung sowie Methoden wie das Elektrospinnen, die Gefriertrocknung und chemische Funktionalisierung weitere Fortschritte bei der Entwicklung künstlicher Implantate versprechen. Es ist zu hoffen, dass mit diesen Ansätzen effiziente zellfreie Implantate hergestellt werden können, die lagerfähig sind und jederzeit vom Neurochirurgen eingesetzt werden können.

\section{Literatur}

Bertleff, M. J., Meek, M. F. und Nicolai, J. P. (2005): A prospective clinical evaluation of biodegradable Neurolac nerve guides for sensory nerve repair in the hand. J Hand Surg Am. 30: 513-518.

Boyd, J. G. und Gordon, T. (2003): Neurotrophic factors and their receptors in axonal regeneration and functional recovery after peripheral nerve injury. Mol Neurobiol. 27: 277-324.

Bozkurt, A., Deumens, R., Beckmann, C., Olde Damink, L., Schugner, F., Heschel, I., Sellhaus, B., Weis, J., Jahnen-Dechent, W., Brook, G. A. und Pallua, N. (2009): In vitro cell alignment obtained with a Schwann cell enriched microstructured nerve guide with longitudinal guidance channels. Biomaterials. 30: $169-179$.

Chew, S. Y., Mi, R., Hoke, A. und Leong, K. W. (2007): Aligned protein-polymer composite fibers enhance nerve regeneration: a potential tissue-engineering platform. Adv Funct Mat. 17: 1288-1296.

Clements, I. P., Kim, Y.-T., English, A. W., Lu, X., Chung, A. und Bellamkonda, R. V. (2009):
Thin-film enhanced nerve guidance channels for peripheral nerve repair. Biomaterials. 30 . 3834-3846.

Dalton, P. D. und Mey, J. (2009): Neural interactions with materials. Frontiers in Bioscience. 14: 769-795.

de Ruiter, G. C., Malessy, M. J., Yaszemski, M. J., Windebank, A. J. und Spinner, R. J. (2009): Designing ideal conduits for peripheral nerve repair. Neurosurg Focus. 26: E5.

Dodla, M. C. und Bellamkonda, R. V. (2008): Differences between the effect of anisotropic and isotropic laminin and nerve growth factor presenting scaffolds on nerve regeneration across long peripheral nerve gaps. Biomaterials. 29: 33-46.

Geiger, B., Bershadsky, A., Pankov, R. und Yamada, K. M. (2001): Extracellular matrix - cytoskeleton crosstalk. Nat Rev Mol Cell Biol. 2: 794-805.

Hadlock, T., Elisseeff, J., Langer, R., Vacanti, J. und Cheney, M. (1998): A tissue-engineered conduit for peripheral nerve repair. Arch Otolaryngol Head Neck Surg. 124: 1081-1086.

Inada, Y., Hosoi, H., Yamashita, A., Morimoto, S., Tatsumi, H., Notazawa, S., Kanemaru, S. und Nakamura, T. (2007): Regeneration of peripheral motor nerve gaps with a polyglycolic acid-collagen tube: technical case report. Neurosurgery. 61: E1105-E1107.

Kawaja, M. D., Boyd, J. G., Smithson, L. J., Jahed, A. und Doucette, R. (2009): Technical strategies to isolate olfactory ensheathing cells for intraspinal implantation. $J$ Neurotrauma. 26: 155-177.

Kim, Y. T., Haftel, V. K., Kumar, S. und Bellamkonda, R. V. (2008): The role of aligned polymer fiber-based constructs in the bridging of long peripheral nerve gaps. Biomaterials. 29: 3117-3127.

Klinkhammer, K., Bockelmann, J., Simitzis, C., Brook, G. A., Grafahrend, D., Groll, J., Möller, M., Mey, J. und Klee, D. (2010): Functionalization of electrospun fibers of poly(epsilon-caprolactone) with star shaped NCO-poly(ethylene glycol)-statpoly(propylene glycol) for neuronal cell guidance. J Mater Sci Mater Med. in press.

Koh, H. S., Yong, T., Chan, C. K. und Ramakrishna, S. (2008): Enhancement of neurite outgrowth using nano-structured scaffolds coupled with laminin. Biomaterials. 29: 3574-3582.

Lietz, M., Dreesmann, L., Hoss, M., Oberhoffner, S. und Schlosshauer, B. (2006): Neuro tissue engineering of glial nerve guides and the impact of different cell types. Biomaterials. 27: 1425-1436.

Matsumoto, K., Ohnishi, K., Kiyotani, T., Sekine, T., Eng, H. U. M., Nakamura, T., Endo, K. und Shimizu, Y. (2000): Peripheral nerve regeneration across an $80-\mathrm{mm}$ gap bridged by a polyglycolic acid (PGA)-collagen tube filled with laminin-coated collagen fibers: a histological and electrophysiological evaluation of regenerated nerves. Brain Res. 868 : 315-328. 
Meek, M. F. und Coert, J. H. (2008): US Food and Drug Administration/Conformit Europeapproved absorbable nerve conduits for clinical repair of peripheral and cranial nerves. Ann Plast Surg. 60: 466-472.

Mohanna, P. N., Young, R. C., Wiberg, M. und Terenghi, G. (2003): A composite poly-hydroxybutyrate-glial growth factor conduit for long nerve gap repairs. $J$ Anat. 203: 553-565.

Schmidt, C. E. und Baier Leach, J. (2003): Neural tissue engineering: strategies for repair and regeneration. Annu Rev Biomed Eng. 5: 293-347.

Schnell, E., Klinkhammer, K., Balzer, S., Brook, G. A., Klee, D., Dalton, P. D. und Mey, J. (2007): Guidance of glial cell migration and axonal growth on electrospun nanofibers of poly-å-caprolactone and a collagen/ poly-å-caprolactone blend. Biomaterials. 28: 3012-3025.

Sulaiman, O. A. R., Boyd, J. G. und Gordon, T. (2005): Axonal regeneration in the peripheral nervous system of mammals. In: Kettenmann, H. und Ransom, B. R. (Hrsg.), Neuroglia. Oxford University Press, Oxford, New York, pp. 454-466.

Wang, W., Itoh, S., Matsuda, A., Aizawa, T., Demura, M., Ichinose, S., Shinomiya, K. und Tanaka, J. (2008): Enhanced nerve regeneration through a bilayered chitosan tube: the ef- fect of introduction of glycine spacer into the CYIGSR sequence. $J$ Biomed Mater Res A. 85: 919-928.

Wang, X., Hu, W., Cao, Y., Yao, J., Hu, J. und Gu, X. (2005): Dog sciatic nerve regeneration across $30 \mathrm{~mm}$ defect bridged by a chitosan/PGA artificial nerve graft. Brain. 128: 1897-1910.

Wangensteen, K. J. und Kalliainen, L. K. (2009): Collagen tube conduits in peripheral nerve repair: a retrospective analysis. $J$ Hand $(N Y)$. doi: 10.10007/s11552-009-9245-0.

Webb, K., Budko, E., Neuberger, T. J., Chen, S., Schachner, M. und Tresco, P. A. (2001): Substrate-bound human recombinant L1 selectively promotes neuronal attachment and outgrowth in the presence of astrocytes and fibroblasts. Biomaterials. 22: 1017-1028.

Weber, R. A., Breidenbach, W. C., Brown, R. E., Jabaley, M. E. und Mass, D. P. (2000): A randomized prospective study of polyglycolic acid conduits for digital nerve reconstruction in humans. Plast Reconstr Surg. 106: 1036-1046.

\section{Kurzbiografien}

Isabell Koxholt: Studium der Geschichte und Biologie an der RWTH Aachen, erstes Staatsexamen 2010; seit 2009 Forschungsprojekt zur Entwicklung künstlicher Implantate für die Nervenregeneration.
Jörg Mey: Studium der Geschichte, Philosophie und Biologie in Freiburg und Tübingen; Auslandsstudium in Kanada, 1987 Bachelor of Science an der Brock University, St.Catharines, Ontario. 1990 Staatsexamen (Lehramt) in den Fächern Biologie und Geschichte. 1994 Promotion an der Universitäts-Augenklinik und am Max-Planck-Institut für Entwicklungsbiologie, Tübingen. 1994-96 Postdoc am Shriver Center/Harvard Medical School, Boston; 1996 Assistent am Institut für Biologie II der RWTH Aachen; 2000 Habilitation im Fach Zoologie; 2001-2003 Vertretungsprofessur für Allgemeine Biologie und Zellbiologie, dann für Zelluläre Neurobionik, seit 2007 außerplanmäßiger Professor.

\section{Korrespondenzadresse}

\section{Jörg Mey}

Institut für Biologie II, RWTH Aachen

Mies-van-der-Rohe-Str. 15

52074 Aachen

Tel.: $\quad+49241-8020826$

Fax: $\quad+49241-8022133$

E-Mail:mey@bio2.rwth-aachen.de

\section{Stipendien für die Göttinger Jahrestagung 2011}

Die Neurowissenschaftliche Gesellschaft e.V. stellt wieder Reisestipendien für die Teilnahme am 9th Göttingen Meeting of the German Neuroscience Society (23.-27. März 2011) zur Verfügung.

Bewerben können sich Doktoranden und junge Postdocs, die max. 35 Jahre alt sind.
Das Reisestipendium in Höhe von 300 Euro wird in bar auf der Tagung ausgezahlt.

Die Bewerbung sollte folgende Unterlagen enthalten:

- einseitiger Lebenslauf

- Publikationsliste

- Kopie des Abstracts

- ein kurzes Empfehlungsschreiben

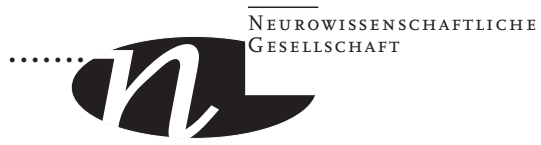

Bewerbungsschluss ist der 15. Oktober 2010.

Die Bewerbung erfolgt über die Website der Neurowissenschaftlichen Gesellschaft e.V. (http://nwg.glia.mdc-berlin.de/de/ stipends/). Postalisch oder per E-Mail eingesandte Bewerbungen werden nicht bearbeitet.

\section{Aufruf zu Kandidatenvorschlägen für die Vorstandswahl der NWG 2011}

Laut Satzung ist im Januar 2011 die Wahl des NWG-Vorstandes für die Amtsperiode 2011-2013, die mit dem Ende der Göttinger Tagung am 27. März 2011 beginnen wird, durchzuführen.

Alle Mitglieder sind aufgefordert, Vorschläge für die Positionen der Sektions- sprecher, des Schatzmeisters, des Generalsekretärs und des Vizepräsidenten bei der Geschäftsstelle per E-Mail (gibson@ mdc-berlin.de) einzureichen.

Das Amt des Präsidenten steht nicht zur Wahl, laut Satzung wird der Vizepräsident der vorangegangenen Amtspe- riode automatisch Präsident der nächsten Amtsperiode.

Der Stichtag für die Einsendung von Vorschlägen ist der 15. September 2010.

Es können nur Vorschläge berücksichtigt werden, die die komplette postalische Adresse, die Telefonnummer und die EMail-Adresse des Kandidaten enthalten. 